\title{
Neue Ordnungsökonomik. Zur Aktualität eines kontextualen Forschungsprogramms: Replik zum Kommentar von Björn Frank
}

\author{
Stefan Kolev • Nils Goldschmidt • Joachim Zweynert
}

Online publiziert: 1. April 2019

(C) List-Gesellschaft e.V. 2019

Der Kommentar von Björn Frank gibt uns die Gelegenheit, auf einige Punkte einzugehen, die offensichtlich der Klärung bedürfen. Dabei fokussieren wir auf die zentralen Punkte, die Frank aufwirft: Forschungsfragen, Methoden und Adressaten der Neuen Ordnungsökonomik.

\section{Wer sind überhaupt die aktuellen Ordnungsökonomen?}

Bevor wir auf diese Fragen Franks eingehen, erscheint eine personelle Klarstellung notwendig. Er nimmt gleich zu Beginn zur Kenntnis, dass die Vertreter der Neuen Ordnungsökonomik durchaus kritisch mit der eigenen Tradition und ihren Versäumnissen umgehen. Gegen Ende seines Abschn. 2 heißt es allerdings, alte wie neue Ordnungsökonomen würden im Vergleich zu anderen Ökonomen eher ,,auf Deutsch statt auf Englisch zu kommunizieren“. Hier gilt es nochmals klarzustellen, wen wir aktuell als Vertreter einer Ordnungsökonomik verstehen. Unser Beitrag führt u.a. Buchanan, Ostrom, North und Sen als Quellen der Inspiration an, aus

This reply refers to the commentary article available online at https://doi.org/10.1007/s41025-01900157-0.

\footnotetext{
S. Kolev $(\bowtie)$

Westsächsische Hochschule Zwickau, Dr.-Friedrichs-Ring 2A, 08056 Zwickau, Deutschland

E-Mail: stefan.kolev@fh-zwickau.de

N. Goldschmidt

Universität Siegen, Kohlbettstr. 17, 57068 Siegen, Deutschland

E-Mail: goldschmidt@wiwi.uni-siegen.de

J. Zweynert

Universität Witten/Herdecke, Alfred-Herrhausen-Str. 50, 58448 Witten, Deutschland

E-Mail: joachim.zweynert@uni-wh.de
} 
denen sich die Neue Ordnungsökonomik speist. Folglich ist es ein größerer Kreis an heutigen Ökonomen, die in diesen Traditionslinien stehen und die wir als Vertreter ordnungsökonomischer bzw. kontextualer Ansätze auffassen, als Franks Einschub zur Kommunikation auf Deutsch suggeriert. Am plastischsten wird es wohl, wenn man auf das Netzwerk für Ordnungsökonomik und Sozialphilosophie NOUS (http:// nous.network) blickt, das wir 2016 mitgegründet haben. Von den momentan über 80 Affiliates des Netzwerkes sind mehr als die Hälfte internationale Forscher auf den Gebieten der Ökonomik, Jura, Politikwissenschaft, Philosophie, Geschichte und Soziologie, die dem Netzwerk beigetreten sind, um die verschiedenen ,,thinking-inorders"-Paradigmen dies- und jenseits des Atlantiks zu revitalisieren und zu verzahnen. Anders als zur Zeit Walter Euckens, ist Deutsch als Kommunikationssprache für ein solches Netzwerk heutzutage doch eher unwahrscheinlich.

\section{Die aktuellen Probleme und die Notwendigkeit eines kontextualen Ansatzes}

Frank beanstandet an mehreren Stellen unsere Behauptung, dass sich die Probleme der heutigen Zeit als „Prozess der kumulativen Ordnungserschütterungen“ begrifflich fassen lassen. Zu den drei Phänomenen, die wir am Anfang unseres Beitrags nennen (Donald Trump, Fragilität der EU, Euro-Krise) merkt er an, dass sich die Euro-Krise doch „orthodox“, „,selbst im Mainstream“ erklären ließe. Das bestreiten wir natürlich nicht, aber gerade dieser Punkt macht deutlich, wo der kontextuale Ansatz möglicherweise im Vorteil ist. Mit „kumulativen Ordnungserschütterungen“ meinen wir, dass die Euro-Krise als Krise der Wirtschaftsordnung mittlerweile in eine deutlich umfassendere gesellschaftliche Krise gemündet ist - die Krise der politischen Ordnung, national wie international. Die politische Fragilität der EU geht inzwischen deutlich über den Brexit hinaus: Die politischen und rechtsstaatlichen Ordnungen zeigen in Ungarn und Polen immer weitere Risse, westliche Werte werden als die Grundlage dieser Ordnungen offen bekämpft, außerdem reißt der neue Dissens in Sachen Migration Gräben auf, die mit fundamentalen Diskussionen über Kultur und Identität einhergehen. All dies macht die Konsensfindung in der EU auch in Sachen Euro - nicht gerade einfacher, wie die aktuellen Entwicklungen in Italien zeigen. Hinzu kommen die Herausforderungen Trumps für die internationalen Beziehungen, der mit seinem „Deals“-Ansatz die globale Ordnung in einen ständigen Stresstest versetzt, bis hin zu den Regelrahmen der WTO und der UN. Neu austarierte Beziehungen zwischen den USA und Russland können auch nochmal neuartige Problemlagen für die EU in Sachen Außen- und Sicherheitspolitik mit sich bringen, abermals mit neuem Bedarf an Konsens, der auch vorher schon durch Euro- und Migrationskrise schwer genug zu finden war. Es ist insbesondere die Interdependenz dieser verschachtelten krisenhaften Phänomene, deren Wesen wir mit „Prozess der kumulativen Ordnungserschütterungen“ für gut abgebildet halten. Und hierbei ist unseres Erachtens das Instrumentarium der isolierenden Ökonomik allein überfordert. 


\section{Methodischer Ansatz der Neuen Ordnungsökonomik}

Die obigen mannigfachen und miteinander verschachtelten Krisen erfordern tiefgründige Kenntnisse nicht nur der Wirtschaftsordnung, sondern unter anderem der Ordnung des Staates, der nationalen, supranationalen und globalen Rechtsordnung, der internationalen Beziehungen und zahlreicher informeller Institutionen wie etwa gesellschaftliche Strukturen und Fragen der religiösen Toleranz. Hier ist nicht nur der isolierende Ökonom überfordert, sondern die Ökonomik schlechthin. Der kontextuale Ansatz ist dahingehend im Vorteil, weil sich die Neue Ordnungsökonomik als Schnittstellendisziplin versteht, welche den Dialog der benötigten Expertisen unterschiedlicher Ordnungen ermöglichen und vertiefen kann.

F.A. Hayek zeigt in seinem Aufsatz „The Dilemma of Specialization“ elegant auf, wie interdisziplinäres Denken auch in Zeiten rasanten wissenschaftlichen Fortschrittes möglich ist (Hayek 1967). Notgedrungen spezialisieren sich heutige Ökonomen auf einzelne Teilbereiche des Faches, das erzwingt die schiere Menge an neuen Ergebnissen in jedem einzelnen Teilbereich. Allerdings müssen diese Spezialisten imstande sein, untereinander und auch zu den Nachbardisziplinen in den Dialog zu treten. Unsere persönliche Erfahrung bei einer Konferenzreihe zum Forschungsprogramm der Neuen Ordnungsökonomik, die wir seit 2009 regelmäßig auf Schloss Ettersburg bei Weimar veranstalten, zeigt deutlich, wie voraussetzungsvoll diese Dialoge sein können - weil die sprachlichen Konventionen, aber auch die verwendeten Methoden so divers sein können. Sobald aber die Brücken zwischen Ökonomen, Juristen, Politikwissenschaftlern, Philosophen, Historikern und Soziologen aufgebaut sind, kann die Methodenvielfalt der einzelnen Spezialisten ausgesprochen fruchtbar genutzt wird.

Frank stellt konkret die Frage, wie sich die Neue Ordnungsökonomik zu empirischen Methoden positioniert. Selbstverständlich haben wir nichts gegen experimentelle oder kliometrische Studien einzuwenden. Ganz im Gegenteil: Vernon Smiths Forschung zeigt eindrücklich, wie Pionierarbeiten zur experimentellen Ökonomik mit fundamentalen Beiträgen zur Ordnungsökonomik wie etwa dem Konzept einer „ecological rationality“ einhergehen können. Wirtschaftsgeschichte - ob kliometrisch wie in den frühen Arbeiten von Donald McCloskey oder ordnungsökonomisch wie in ihren jüngsten Beiträgen - können selbstverständlich erhellend für das Studium der Ko-Evolution verschiedener gesellschaftlicher Teilordnungen sein. Und natürlich ist die Schätzung von Regressionskoeffizienten nicht ,,,isolierend“ per se“, um Franks Frage zu beantworten. Ordnungsökonomen haben allerdings einen weiten Empirie-Begriff: Dazu gehören die obigen Ansätze, aber auch das qualitative Studium von Wirtschafts- und Sozialgeschichte, die wir je nach Erkenntnisziel für ähnlich wertvoll halten wie quantitative Ansätze (McCloskey 2005) und ja auch im Mainstream ihren Platz haben, wie der Bestseller „Why Nations Fail“ gezeigt hat. Die Geschichtswissenschaft zeigt in ihrer ganzen Breite, dass die von Frank eingeforderte ,präzise Hypothesenentwicklung“ auch mit klarer verbaler Logik erreichbar ist und nicht nur mit quantitativen Methoden. Die für Ordnungsökonomen so wichtige Geschichte des ökonomischen Denkens verdeutlicht, dass das Gros der Erkenntnisse unseres Faches aus Epochen stammt, als qualitatives Argumentieren mit verbaler Logik ein zentrales Erkenntnisinstrument war. Es wäre gerade für die 
öffentliche Debatte heute wohl wünschenswert, wenn die Genauigkeit des Arguments für Ökonomen die gleiche Bedeutung besäße wie die formale Genauigkeit empirischer Studien. Und überhaupt: Eine solche methodologische bzw. erkenntnistheoretische Diskussion, kritisch über die Beziehung zwischen Sozialwissenschaften, Philosophie und Ökonomik zu reflektieren, gehört zum genuinen Anspruch der Ordnungsökonomik - was wohl nicht immer auf andere Teildisziplinen der Wirtschaftswissenschaft zutrifft.

\section{Adressat der Neuen Ordnungsökonomik}

Dass ein wissenschaftlicher Text wie unserer ,,den Bürger“ weder berührt noch interessiert“, ist durchaus möglich, je nachdem, welchen epistemischen Horizont Frank dem Bürger zutraut. Dass die Ordnungsfragen von Wirtschaft und Gesellschaft den Bürger kaum interessieren, halten wir für weniger wahrscheinlich. Es ist polemisch, wenn Hayeks „Road to Serfdom“ von Frank als der ,letzte ordnungsökonomische Bestseller“ hingestellt wird: In den letzten Jahrzehnten sind verschiedene publikumswirksame Werke erschienen, die in unserer oben nochmals erläuterten Lesart als Beiträge zur Ordnungsökonomik zu sehen sind. Das ist aber auch nicht der zentrale Punkt, wenn wir schreiben, dass Bürgerberatung ein Hauptziel von Ordnungsökonomen sein soll. Zum einen wollen wir darauf hinweisen, dass Ökonomen - in der Formulierung Herbert Gierschs - überhaupt eine Bringschuld gegenüber der Gesellschaft haben (Horn 2008; Barbier 2013), dass also im Wissenschaftsbetrieb auch Aktivitäten jenseits der Publikationen in Fachzeitschriften eine Wertschätzung erfahren sollten. Zum anderen hat Susanne Cassel - auf der Grundlage der zentralen Arbeiten von Viktor Vanberg - klar herausgearbeitet, warum Bürger- und nicht Politikerberatung zielführend sein kann, diese Bringschuld zu erbringen (Cassel 2004). $\mathrm{Ob}$ dies in Blogs und sozialen Medien passiert, in klassischen Medien oder durch die Präsenz in der Zivilgesellschaft, sei jedem einzelnen und seinem komparativen Vorteil überlassen. Deirdre McCloskeys Plädoyer für - auch von Ökonomen oft unterschätzte - Achtsamkeit bei der Wahl der Rhetorik (McCloskey 1985) ist in den heutigen aufgeheizten Debatten umso mehr zu beherzigen.

In einer vom „Prozess der kumulativen Ordnungserschütterungen“ geprägten Welt - und das bleibt unsere Diagnose - muss die Ökonomenzunft den Bürger wieder von ihrer umfassenden Ordnungsexpertise überzeugen, nur so kann sie zur Therapie beitragen. Dass das noch ein weiter Weg ist, damit sind wir uns mit Frank völlig einig.

\section{Literatur}

Barbier, H. D. (2013). Die Bringschuld des Ökonomen als Verpflichtung und Befreiung. In L.P. Feld, K. Horn \& K.-H. Paqué (Hrsg.), Das Zeitalter von Herbert Giersch. Wirtschaftspolitik für eine offene Welt (S. 3-7). Tübingen: Mohr Siebeck.

Cassel, S. (2004). Politikberatung und Politikerberatung: Eine institutionenökonomische Analyse der wissenschaftlichen Beratung der Wirtschaftspolitik (2. Aufl.). Bern: Paul Haupt.

Hayek, F. A. v. (1967). The dilemma of specialization. In Studies in philosophy, politics and economics (S. 122-132). London: Routledge. 
Horn, K. (2008). Der Bankrott der Ökonomen. Die Finanzkrise enthüllt auch das Versagen der Wirtschaftswissenschaften. Internationale Politik, 15(12), 54-55.

McCloskey, D. N. (1985). The rhetoric of economics. Madison: University of Wisconsin Press.

McCloskey, D. N. (2005). The trouble with mathematics and statistics in economics. History of Economic Ideas, 13(3), 85-102. 